\title{
Ultra Wide Band Positioning Systems for Advanced Construction Site Management
}

\author{
Alberto Giretti, Alessandro Carbonari and Massimo Vaccarini
}

Additional information is available at the end of the chapter

http://dx.doi.org/10.5772/48260

\section{Introduction}

Phenomena of scarce performances and rapid obsolescence of buildings, entailing high maintenance costs, are very often the consequence of a loose integration between the operational phases of the building process. The separation of technical and management competences, the lack of coordination between high level strategic and procurement decisions and on-site construction management, make the achievement of designed performances a particularly fragile process and it is one of the side effects of a number of the singular complexities of the building construction process. Nearly every facility is, in fact, custom designed and nomadic. Construction sites are very dynamic working environments, often changing their layout on a daily or weekly basis. They are affected by unpredictable and uncontrollable external events such as weather, the availability of local resources, etc. (Behzadan et al. 2008). Furthermore, large building projects involve thousands of parts and components while changes to design plans at construction time are not uncommon. Building parts and components are mostly made or assembled on-site, standardization is rather low. Adjustments are also made on site, at times without even updating original building plans. Consequently, the management of building construction is a rather complex task. The high investments normally required for construction facilities call for the optimization of workflow efficiency and of the related financial effort. On the Health \& Safety side, workers' safeguard in construction sites is always among project managers' major concerns. The complexity of on-site working conditions requires the careful planning and coordination of several crews and equipment in order to ensure safety. The optimization of safe working conditions places many constraints on: mobilization, transportation, collaboration of equipment, work interference, tight schedules and spatial constraints. The traditional programmatic approach, based on a careful but a-priori planning of the working conditions and on the preliminary identification of the critical conditions, is not capable of coping effectively with the high dynamic nature of the 
construction site, affected by frequent rescheduling and repositioning of the working activities. In this operational context, support to the management of critical performance factors such as production progress, production quality and workers' safety requires the implementation of very flexible and effective real-time monitoring systems: systems capable of accurately tracking workers, goods and the position of equipment so that workflow information can be provided to the programming/control department in real-time and critical, hazardous situations can be predicted. The harsh, highly dynamic nature of the construction environment calls for position tracking systems capable of working without line of sight, systems accurate enough to track assets in real-time with an error comparable with a worker's footprint (about $0.60 \mathrm{~m}$ ) with a signal ranges of hundreds meters, and whose deployment does not cause high impact and costs to the layout management of construction sites, while requiring low maintenance.

Recently, a set of new low cost and highly standardized technologies, like Global Positioning Systems (GPS), Wireless Networks, Radio Frequency Identification (RFID), the IEEE 802.15.4 (ZigbBee) communication protocol, etc. provided the technological basis for the dynamic position tracking of workers and materials, allowing the development of innovative real-time operational and management services for highly automated nomadic construction facilities (Fontana \& Gunderson 2002), (Khoury \& Kamat 2009). Position tracking is achieved by establishing a referenced radio frequency environment, made up of a series of transmitters/receivers nodes, and by assigning a sensor to each tracked entity. The sensor is capable of receiving/transmitting signals from/to the reference environment. Locating a node in these wireless environments therefore involves the collection of location information from radio signals traveling between the target and the reference nodes. Radio signal's information regarding the angle of arrival (AOA), the signal strength (SS), or time of arrival (TOA) have been used to determine the location of the target node (Caffery 2000). The AOA-based positioning technique involves measuring angles of the target node seen by the reference nodes, which is done by means of antenna arrays. To determine the location of a node in a two-dimensional (2-D) space, it is sufficient to measure the angles of the straight lines connecting the node and two reference nodes. The AOA approach is not well suited for position tracking in construction sites because AOA information in the non-line-of-sight situation rapidly becomes a major error source in positioning and tracking. SS relies on a path-loss model. The distance between two nodes can be calculated by measuring the energy of the received signal at one node. This distance-based technique requires at least three reference nodes to determine the 2-D location of a given node, using triangulation (Caffery 2000). Unfortunately, SS-based positioning algorithms are very sensitive to the characteristics of the channel. The variance of a distance estimation obtained from SS measurements is lower bounded by the Cramér-Rao lower bound (CRLB) (Qi \& Kobayashi 2003):

$$
\sqrt{\operatorname{Var}(\hat{d})} \geq \frac{\ln 10}{10} \cdot \frac{\sigma_{s h}}{n_{p}} \cdot \hat{d}
$$


where $\hat{\mathrm{d}}$ is the distance between the two nodes, $n_{p}$ is the path loss factor, and $\sigma_{\text {sh }}$ is the standard deviation of the zero mean Gaussian random variable representing the log-normal channel shadowing effect (where a large obstruction obscures the main signal path between transmitter and receiver). In general, since the best achievable limit depends on the channel parameters and the distance between the two nodes, the highly dynamic nature of constructions sites hinders precise control of the positioning accuracy. Nevertheless, in cases where the target node can be kept close to one or more reference nodes, the SS measurements may provide enough precision for practical purposes (Sahinoglu \& Catovic 2004). In fact some applications of the SS method based on ZigBee have been developed for real-time construction management (Carbonari et al. 2010). In these cases the system is designed in such a way that the reference radio frequency frame overlays a rather tight zoning over the working environment, so that, wherever the target node is positioned, it is in proximity of one or more reference nodes. SS localization is performed through the application of the "Weighted Centroid Localization" (Blumenthal et al. 2007), according to which the position of the target node is computed as a weighted average value of the positions of the routers closest to the tag. Technically, a mobile node who wants to evaluate its position sends a broadcast message to all the reference nodes in the network in its radio range. Each router sends its PAN (Personal Area Network) coordinator a short message whose payload contains its fixed coordinates and the message's RSSI (Received Signal Strength Indication), measured in $\mathrm{dBm}$. The RSSI is then used by a central application server, directly connected to the PAN coordinator via TCP/IP, to estimate the weighting coefficient associated to each router's coordinates for implementing the Weighted Centroid Localization algorithm. TOA positioning techniques rely on measurements of the travel times of signals between nodes. If two nodes have a common clock, the node receiving the signal can determine the time of arrival (TOA) of the incoming signal that is time-stamped by the reference node. The Global Positioning System (GPS) (Ergen et al. 2007) was one of the early systems providing TOA based position tracking support to construction management applications. GPS is a spacebased satellite navigation system that provides location and time information in all weather, anywhere on or near the Earth, where there is an unobstructed line of sight to four or more GPS satellites. A GPS receiver calculates its position by precisely timing the signals sent by GPS satellites high above the Earth. Each satellite continually transmits messages that include the time the message was transmitted and the satellite position at the time of the message transmission. The receiver uses the messages it receives to determine the transit time of each message and computes the distance to each satellite. The application of the GPS technology for position tracking on construction sites is severely limited by the non-line-of-sight (NLOS) occurring in indoor environments. Nevertheless, within this limit, a number of construction management applications, concerning outdoor activities, built by integrating GPS position data with the information embedded into Radio Frequency Identifiers (RFID) have been proposed in the last decade. Integrated RFID and GPS technology have been developed for the purpose of: tracking highly customized prefabricated components, avoiding delays in construction (Ergen et al. 2007); embedding RFID tags in building components to store design data, which can be passed to the people in charge of maintenance during the operational phase (Cheng et al. 2007) and improving the efficiency of tool tracking and availability by using RFID tags (Gajamani \& Varghese 2007). The FutureHome EU funded project 
(Abderrahim et al. 2005) has developed systems for product and process analysis suited to manufactured and prefabricated construction solutions. The advantages conveyed by mobile computing in construction management have also been described in (Rebolj et al. 2001), the most relevant ones being: information embedding; supply delivery records and progress updates directly at the jobsite; access to as-built and up-to-date documents; rapid communication and collaboration throughout the entire project life cycle. On the Health \& Safety management side, (Abderrahim et al. 2005) propose a mechatronic helmet, using a GPS antenna and a bidirectional communication system, for workers' safety control. The helmet is capable of position tracking and is integrated in a computer based control system that implements a rather articulated safety control policy. (Wang et al. 2004) developed a policy for collision detection among construction equipment. The role of handheld and wearable computing has also been widely investigated (Fuller et al. 2002).

\section{Ultra Wide Band geolocation systems}

Ultra Wide Band (UWB) is a mature TOA geolocation technology that has undergone considerable development in the last decades.

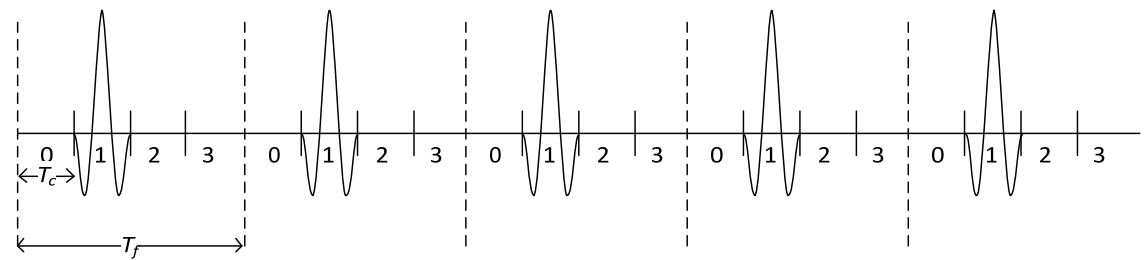

Figure 1. A sample transmitted signal from a time-hopping impulse radio UWB system. $\mathrm{T}_{\mathrm{f}}$ is the frame time and $T_{c}$ is the chip interval.

The majority of the initial concepts and patents for ultra-wideband (UWB) technology originated in the late 1960s at the Sperry Research Center (Sudbury, MA), then part of the Sperry Rand Corporation, under the direction of Dr. Gerald F. Ross. UWB is a technology for transmitting information spread over large bandwidth $(>500 \mathrm{MHz})$ while allowing spectrum sharing with other users. It is based on the transmission of a baseband impulse, made of a few cycles of an RF carrier, with low duty cycles resulting in a very low power spectral density, as typically produced by impulse or step-excited antennas (Figure 1). The main advantages in using UWB for geolocation are the absence of steady waves generated by obstacles and the immunity w.r.t frequency selective effects. Denoting with $c$ the speed of light, with SNR the signal-to-noise ratio, with $S(f)$ the Fourier transform of the transmitted signal and with $\beta$ the effective (or root mean square) signal bandwidth defined by:

$$
\beta \equiv\left[\frac{\int_{-\infty}^{\infty} f^{2}|S(f)|^{2} d f}{\int_{-\infty}^{\infty}|S(f)|^{2} d f}\right]^{\frac{1}{2}}
$$


a distance $\widehat{d}$ between two nodes obtained from TOA estimation when a single-path additive white Gaussian noise (AWGN) channel is assumed, is subject to the following lower limit for the achievable accuracy (Poor 1994), (Cook \& Bernefeld 1970):

$$
\sqrt{\operatorname{Var}(\hat{d})} \geq \frac{c}{2 \sqrt{2} \sqrt{S N R} \beta^{\prime}}
$$

Equation (3) suggests to increase SNR or the effective signal bandwidth for improving accuracy of a TOA based approach: this implies that the use of very large bandwidths (such as in UWB radios) allows, at least in principle, extremely accurate location estimates. For example, for a received UWB pulse of $1.5 \mathrm{GHz}$ bandwidth with $\mathrm{SNR}=0 \mathrm{~dB}$ the lower limit for accuracy falls below one inch. In order to achieve these theoretical estimation accuracies, clock synchronization and clock jitter between the nodes become key factors (Shimizu \& Sanada 2003). Time-difference-of-arrival (TDOA) technique (Caffery 2000) can be employed in order to relax the need for synchronization between a given node and the reference nodes, but synchronization among the reference nodes is always required. By estimating the TDOA of two signals traveling between the given node and two reference nodes, the actual location of the node is restricted on a hyperbola, with foci at the two reference nodes. Therefore, a third reference node is needed for localization on a two dimensional space.

A typical UWB geolocation system is made of one or more tags and of multiple UWB daisychained (usually with CAT-5 cables) beacons, that relay processed time-of-arrival data. A UWB Tag sends out sequence of packet bursts. The frequency-locked UWB receivers measure times-of-arrival and a central processor accurately estimates tag position from the set of differential times-of-arrival by minimizing an error functional. Given $N$ reference nodes with locations $\theta_{i}, i=1, \ldots, N$, a node located in unknown location $\theta$ and a number of $N$ TOA measurements $\tau_{i}, i=1, \ldots, N$ with reliability described by scalar weighting factors $w_{i}$, $i=1, \ldots, N$ and defining the distance $d_{i}(\theta)=\theta-\theta_{i}$ between the given node and the $i^{\text {th }}$ reference node, the following low-complexity least squares (LS) approach can be used to formulate the error functional whose minima is the node location estimate (Caffery 2000):

$$
\hat{\vartheta}=\arg \min _{\vartheta} \sum_{i=1}^{N} \omega_{i}\left[\tau_{i}-d_{i}(\vartheta) / c\right]^{2}
$$

In practical UWB systems, the TOA is usually detected using a correlation-based estimation algorithm that calculates the time at which a matched filter output peaks. In principle, especially in indoor installations, the detected TOA may not be the true TOA since multiple replicas of the transmitted signal, due to multipath propagation, may partially overlap and shift the position of the correlation peak. Due to the large bandwidth of the UWB signal, multipath components are usually resolvable without the use of complex algorithms, since the detection of the first arriving signal path is usually possible with the necessary accuracy (Gezici et al. 2005). 


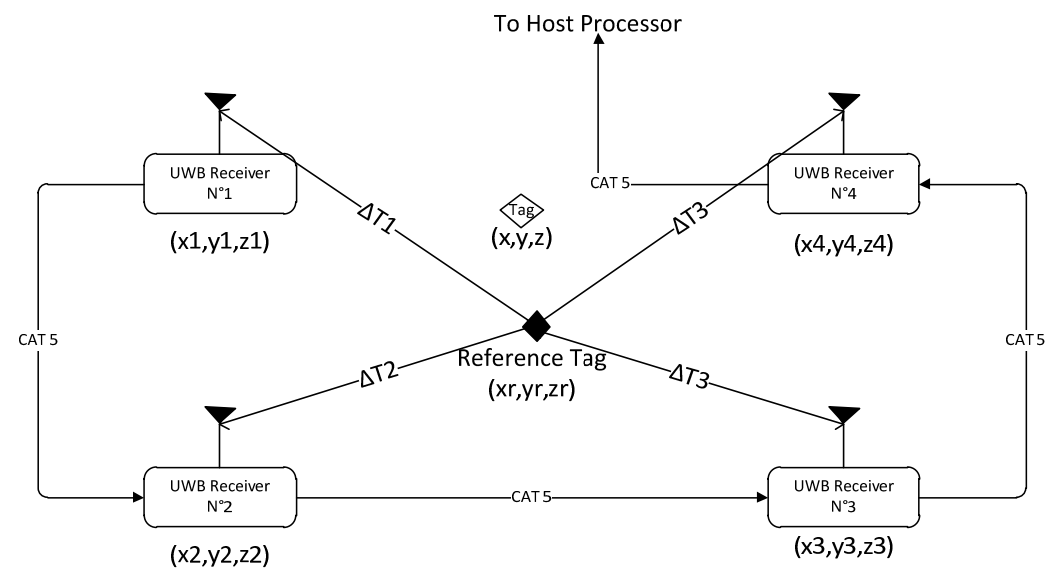

Figure 2. A typical UWB configuration

A second source of position estimation error is non-line-of-sight (NLOS) occurrence between the transmitter and the receiver which occurs when the direct line-of-sight (LOS) between two nodes is completely blocked. In these cases only reflections of the UWB pulse reach the receiving node and the delay of the first arriving pulse is always bigger than the true TOA: the extra distance traveled by the pulse generates a positive bias in the measured time delay called NLOS error. Since, LS technique in (4) gives the optimal results in terms of Maximum Likelihood Estimate (MLE) provided that the measurement error is a zero mean Gaussian random variable with known variance, a positive bias would produce large errors in the location estimation. Additional positive bias is introduced when (especially in NLOS propagation) the first arriving pulse is not the strongest pulse and conventional TOA estimation method select the strongest path for estimating TOA. A unified analysis of the NLOS location estimation problem is introduced in (Gezici et al. 2005) where asymptotically optimal estimators (even in presence of statistical NLOS information) are presented. For UWB systems it is shown that the first arriving signals from the LOS nodes can be used to get an asymptotically optimal receiver performance. Figure 3a shows a simple location estimation scenario, where six reference nodes are trying to locate the target node in the middle, and figure $3 \mathrm{~b}$ shows the minimum positioning error versus bandwidth for different number of NLOS nodes (Gezici et al. 2005).

In the optimal case, the high bandwidth allows UWB to theoretically provide position accuracy below $30 \mathrm{~cm}$. Nevertheless, the required presence of at least one LOS receiver and the fact that, for a given installation, the number of NLOS receiver significantly affects the system position estimation accuracy, opens the floor to further investigations concerning the performance of UWB position tracking systems in real working environments. At present only few analyses have been carried out concerning the application of UWB to the construction sector. The following sections report on practical applications of UWB in real world construction environments and further discusses their implications for the development of work progress and health \& safety management. 


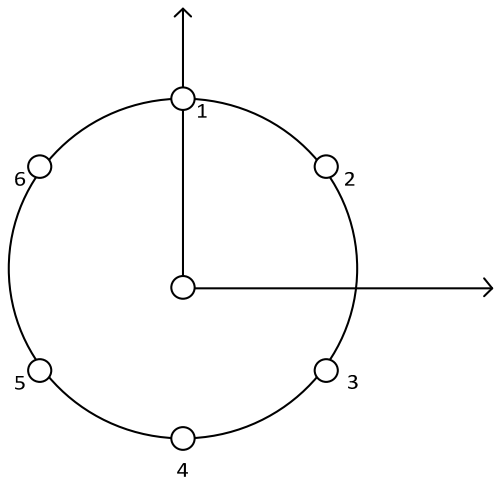

(a)

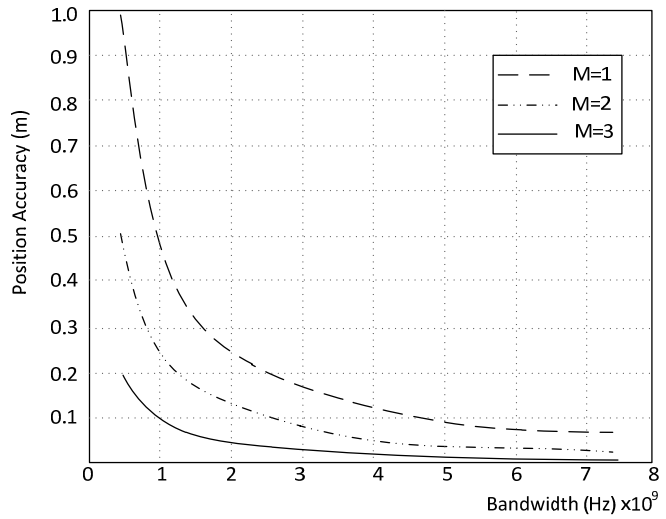

(b)

Figure 3. (a) A simple location estimation scenario where the target node is in the middle of six reference nodes located uniformly around a circle. (b) The minimum positioning error plotted against the effective bandwidth for different numbers of NLOS nodes, $\mathrm{M}$, at $\mathrm{SNR}=0 \mathrm{~dB}$. For $\mathrm{M}=1$, node 1 ; for $\mathrm{M}=2$, node 1 and 2; and for $\mathrm{M}=3$, node 1, 2, and 3 are the NLOS nodes.

\section{UWB position tracking in building construction sites}

The application of UWB technology to construction sites has both practical and technological implications. From the practical standpoint the main issue is the operational impact on site layout. UWB needs CAT5 cables to link receivers to central units in order to transmit data and to keep them in sync. Laying CAT5 cables on a construction site is usually a tricky task because the harsh and continuously varying environment imposes having to place receivers along boundaries, minimizing crossing paths as much as possible. This can be a severe constraint on the design of UWB tracking systems concerning the minimization of NLOS paths between receivers and tags. Furthermore, TDOA systems require one or more reference tags, used only for sync purposes, to be placed in locations with at least 3 LOS paths to as many receivers. The more the construction progresses, the more likely it is that this condition will be violated, requiring a reconfiguration of the UWB system. On the technological side, the main issues concerning the application of UWB position tracking to construction sites are the operational range, the accuracy and the reliability of the position tracking of material/workers proceeding at usual transport/walking speed through eventually obstructed NLOS conditions. An operational range between $50-70 \mathrm{~m}$ is usually required to cover a typical medium dimensioned construction site with a reasonably sized receiver set-up where, for example, typical medium sized site corresponds to blocks of flats or medium public building. The operational range of an UWB system is related to signal attenuation depending on the distance from the receivers, on the number of interfering obstacles, and on the power of the tags. Commercial systems offer tags with power ranging from $300 \mathrm{~mW}$ to $1 \mathrm{~W}$. On-field experiments have demonstrated that high power tags are required when signal attenuation occurs; like those caused by building envelopes and 
internal wall partitions. On the other hand, low power tags can be used in the early phases of building construction, when work is typically carried out in large open areas. Since these two distinct operating conditions substantially affect, as we will later demonstrate, both operational range and tracking accuracy, a fundamental guideline for UWB system design in construction site emerges. The UWB set-up should be arranged in two different installation phases. In an early construction phase (which typically encompasses the site setup, the excavation, the foundation and the elevation of the frame structure) a boundary receiver lay-out is sufficient to accurately trace workers, equipment and material position. A second phase, starting from the construction of the envelope walls, requires one or more complementary UWB systems to be laid down in the scaffolding boundary, i.e. possibly using the scaffold structure to hang receivers. The scaffold structure can be used to trace workers on different floors as well, implementing a 3D receiver configuration. Multi-floor slab paths should be minimized in this case. Figure 4 illustrates an example of the two UWB set-up phases proposed for a typical construction made up of two blocks of flats. Concerning the early construction phases, (Cheng et al. 2011) reports about an accurate assessment of UWB error ranges tracking worker positions in a $2400 \mathrm{~m}^{2}$ construction pit scenario, and in a large $64000 \mathrm{~m}^{2}$ lay down yard. In the construction pit case, the receiver configuration diameter was $65 \mathrm{~m}$ and in the lay down yard, the installation diameter was $270 \mathrm{~m}$. The registered errors are approximately $0.4 \mathrm{~m}$ and approximately $1.5 \mathrm{~m}$ for the construction pit and the lay down yard set-ups, respectively. These results show quite clearly that in the initial excavation, foundation and frame elevation phases UWB technology can be successfully applied for real-time tracking of workers and material. To extend these results to the following phases of the building construction, when envelope and partition walls are elevated, the degradation of the tracking accuracy must be assessed, since in these cases the minimum number of receivers (i.e. three for 2D tracking, four for 3D tracking) with LOS transmission path may fail to occur.

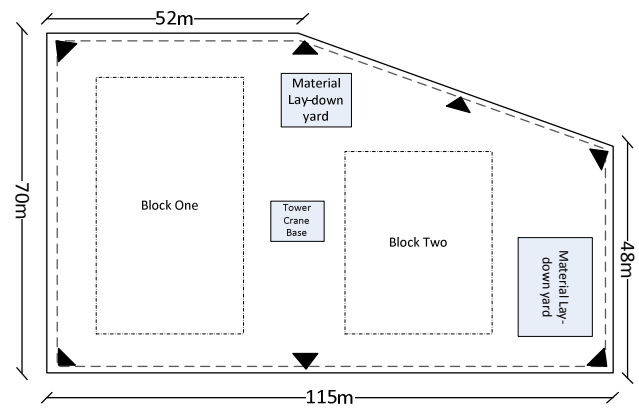

(a)

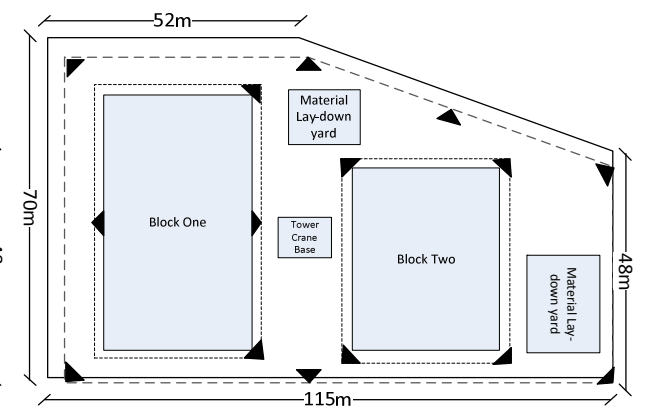

(b)

Figure 4. Typical UWB plant layouts in different construction phases. (a) Boundary layout is sufficient to trace workers and materials during the early phases. (b) Complementary UWB systems should be laid down to track works in advanced construction phases. Black triangles represent receivers. 
We will further detail this noteworthy operational condition later in this section. Figure 5 shows a typical construction site in an advanced construction phase, immediately following the completion of the reinforced concrete frame and floor slabs (a), and after the completion of the envelope walls and internal partition walls (b). During these phases the work frequently moves from buildings' exterior to their interior, with a great deal of activities performed on scaffolding. Hence, the signal quality provided from inside a framed building and from workers moving on scaffolding to a boundary positioned receiver must be assessed. The authors carried out a number of experiments to test these specific operational conditions. The experiments were conducted on a block of flats (Figure 5) built with a reinforced concrete frame structure and light masonry walls. External hollow walls including $0.05 \mathrm{~m}$ polystyrene insulation, as in Figure $6 \mathrm{a}$, had the external wall layer of solid bricks and the internal one of cellular $0.08 \mathrm{~m}$ blocks. Partitioning walls between apartments were made of $0.12 \mathrm{~m}$ thick concrete cellular blocks (Figure $6 \mathrm{~b}$ ) while the walls between rooms of the same apartment were made of $0.08 \mathrm{~m}$ thick cellular blocks (Figure 6c). In order to capture the effects of the envelopes and those of the partition walls, tests were performed just after the completion of the concrete frame structure (Figure 5a) and after the completion of the walls (Figure 5b). The first set of measurements was conducted just after the completion of the reinforced concrete frame and floor slabs. The $0.3 \mathrm{~W}$ tag was subject to blinking in this configuration and therefore considered quite unreliable while the $1 \mathrm{~W}$ power tag behaved consistently.

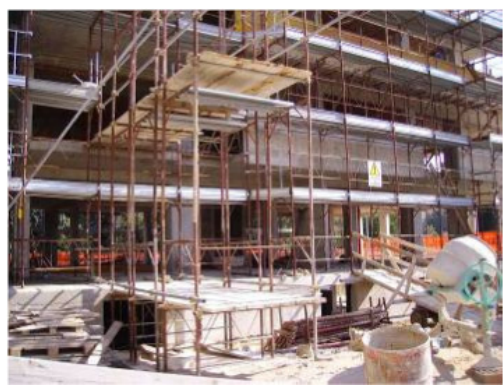

(a)

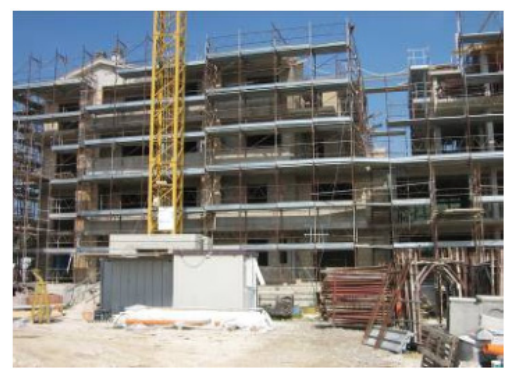

(b)

Figure 5. Typical conditions of a construction site in an advanced construction phase, (a) just after the structural frame completion and (b) after the construction of the envelope and of the internal partitions.
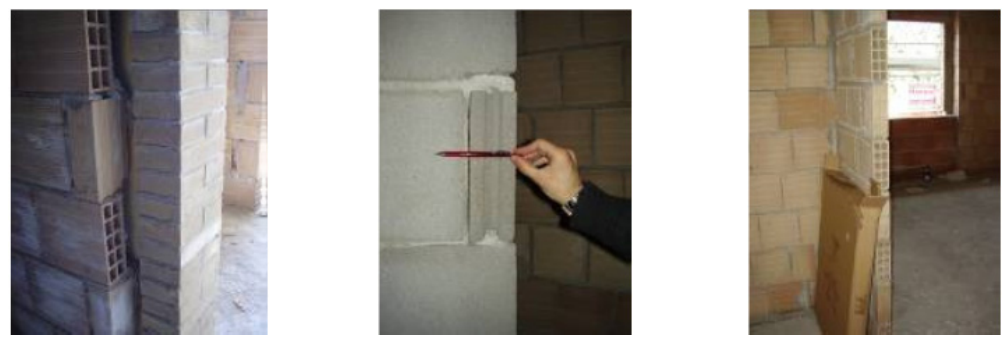

Figure 6. Building technologies used for the envelope and the external partitions in the test building. 
Figure 7 shows the scheme of the UWB setup used for these tests: four receivers were placed at the corners of the site (4 m high) and one reference tag in the center of the building's floor at a height of $1.20 \mathrm{~m}$. It is worth noticing that a metallic scaffold was installed along the building's perimeter. The entire measurement area was approximately rectangular and measured 38x35 m.

A worker moving about on the building ground slab and a worker moving on the scaffold were tracked on predetermined paths, and the error was calculated by comparing the workers' actual routes with the ones detected by the system. In general, the UWB system was able to track the workers' position both on the buildings' ground floor and when moving along the front scaffold, at different heights, with an average position error of about $0.4 \mathrm{~m}$, accordingly to the findings, in the early phases, reported by (Cheng et al. 2011). Analogous 2D measures were made on the first and second floor paths resulting that the receivers were able to track the worker up to the moment when the path height did not overcome the receiver height (second floor), being the receivers pointed slightly downward.

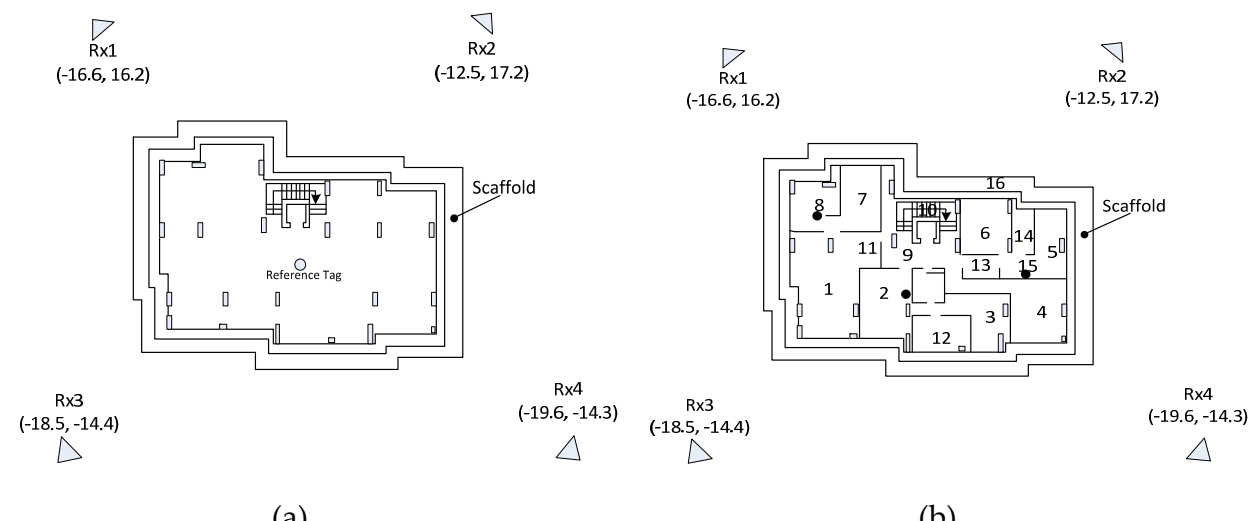

(a)

(b)

Figure 7. Lay-out of the UWB setup used for testing advanced construction phases. In both cases four receivers were placed at the corners of the construction site $(4 \mathrm{~m}$ high). (a) Configuration after the structural frame completion. One reference tag was placed in the center of the building's floor at a height of $1.20 \mathrm{~m}$. (b) Configuration after the completion of the envelope and of the internal partition walls. Two more reference tags were used to enhance tracking reliability.

However, only the $1 \mathrm{~W}$ tag worked well, the other signal $(0.3 \mathrm{~W})$ demonstrated that it was not strong enough. Once the envelopes and internal partition walls were completed, a second measurement set was carried out. In this case the UWB system boundary set-up, as shown in Figure $7 \mathrm{~b}$, did not allow for real-time tracking of workers' positions. In fact, one or more receivers failed to obtain the signals from some locations inside the building from Tag $1 \mathrm{~W}$, hence indicating that walls act as obstacles. A number of systematic field tests were performed, encompassing 16 different positions on the first floor, as shown in Figure $7 \mathrm{~b}$. The results are listed in Table 1, indicating the receivers capable of getting the signal and the tracking quality for each position. For the positions identified by the "M0" type error, the tag was incapable of pointing 3 receivers at the same time. Given that three receivers are 
always visible, we can then infer that the frequency of the received signal was too low (low quality), due to obstacles. By comparing Table 1 with Figure $7 \mathrm{~b}$, relative to the walls' completion stage at the site, it is possible to infer general rules about the behavior of UWB systems in typical reinforced concrete frame constructions. Single layer walls made up of $0.08 \mathrm{~m}$ cellular blocks are quite transparent to UWB. Hollow walls having a double layer of blocks and internal insulation, and concrete cellular blocks both weaken UWB signals. For example, signals travel from position no. 9 to receiver 1, but not from position no. 6 to receiver 1: signal is blocked by the hollow wall between positions no. 9 and 6 . A similar statement holds true for the cellular concrete block wall between positions no. 11 and 2 . When three receivers are in the line-of-sight, then localization works properly (e.g. in positions no. $4,10,11)$. Instead, it could not work, even if three receivers are read, in the event that the quality of the signal received is low (such as in cases no. 5, 6, 16, 17).

\begin{tabular}{|c|c|c|c|}
\hline Position & Receivers & Result & Errors \\
\hline 1 & 1,4 & bad & M0 \\
\hline 2 & 1 & - & - \\
\hline 3 & 1,2 & - & - \\
\hline 4 & $1,2,3$ & good & Ref750A \\
\hline 5 & $2,3,4$ & bad & M0 \\
\hline 6 & $2,3,4$ & bad & M0, R0 \\
\hline 7 & $1,3,4$ & blinking & M0 \\
\hline 8 & $1,3,4$ & discrete & M0 (rare) \\
\hline 9 & $1,3,4$ & discrete & M0 (rare) \\
\hline 10 & $1,3,4$ & good & No \\
\hline 11 & $1,3,4$ & good & No \\
\hline 12 & 1,2 & bad & M0 \\
\hline 13 & 2,3 & bad & M0 \\
\hline 14 & 3,4 & bad & M0 \\
\hline 15 & 2,3 & bad & M0 \\
\hline 16 & $1,3,4$ & bad & M0 \\
\hline
\end{tabular}

Table 1. Tracking quality in different locations.

In this case, the number of receivers used in relation to the complexity of the environment is too low. In general, UWB demonstrated to be able to pass through a maximum of two heavy walls. Therefore, in the later building construction stages, denser and closer receiver configuration, such as those depicted in figure $4 b$, are required. Further tests were carried out in a smaller area in order to simulate the improved configuration. The tests were done using the system setup of figure $7 \mathrm{~b}$ but with receivers no. 2 and no. 3 moved to the right, up to positions $(-2.1,-14.3)$ and $(-2.1,-17.2)$ respectively. This configuration significantly reduced the receivers' distance and the tracking area, resembling the scaffold position of the 
receiver recommended at the beginning of this section. The resulting tracked area was limited to positions 1, 2, 7, 8 and 11. The results reported in Table 2, show that, with the exception of position no.1 (that is in contrast with Table 1, hence considered as an anomaly), the tag's signal was always received by 3 receivers, providing a significant increase in the quality of tracking. Summarizing, different results were obtained for the two construction phases identified. During the site set-up, the excavation, right through to the elevation of the frame, an accuracy of about $0.3-0.4 \mathrm{~m}$ can easily be obtained. Therefore, up until the reinforced concrete frame structure is constructed, four receivers proved to be sufficient for monitoring the workers' movement over the entire site on the ground floor and on the scaffold. In the later construction progress phase, after the elevation of the walls, the situation changed significantly.

\begin{tabular}{|c|c|c|c|}
\hline Position & Receivers & Results & Errors \\
\hline 1 & 1,2 & bad & M0 \\
\hline 2 & $1,2,3$ & Discrete & M0 (rare) \\
\hline 7 & $1,3,4$ & Discrete & M0 (frequent) \\
\hline 8 & $1,3,4$ & Discrete & M0 (frequent) \\
\hline 11 & $1,3,4$ & Discrete & M0 (frequent) \\
\hline
\end{tabular}

Table 2. Tracking quality after the relocation of the receivers.

In this case, the UWB system should be reconfigured to ensure sufficient accuracy for tracking workers reliably. Preliminary results demonstrated that a complementary scaffolding set-up can be used to enhance position tracking to support practical applications. In the following two sections we will point out how the results discussed in this section may be used to develop supporting systems in two main operational applications of construction management: health \& safety and work progress tracking.

\section{Proactive hazard detection}

Notwithstanding the fact that fatalities in construction sites have recorded a decreasing trend in the European area, the construction industry still holds the poorest Health and Safety (H\&S) record of any major industry, where the probability of construction workers being killed is higher than the average for all industries in Europe (European Commission 2008). Such statistics show that the major types of accidents are the result of fatal falls to a lower level, the falling of objects and collisions with means of transport and mobile plants. Traditional practices such as rewarding, training or feedback communication have been shown to be only partially effective on construction sites, as they are unstructured work places. Indeed, they are rendered more complex by the presence of different technologies, requiring the use of several types of equipment and resources at the same time. For this reason, the occurrence of accidents can be only predicted with difficulty and each site has its own peculiarities. As a consequence, much research has been devoted to the development of 
intelligent control systems which adopt advanced communication technologies as the means for establishing new automated control systems. The approach to intelligent support for health \& safety management presently follows two main guidelines. On one hand, automation is applied to support the procedures that current European legislation and many other member countries impose to plan health \& safety measures at design time, in order to discern all the procedures that need to be adopted in order to avoid accidents, according to each site's expected work schedule. Since real work schedule in the execution phase is generally different from the planned one, and since plan updates, collected during regular inspections, require a certain time to be implemented, work monitoring facilities capable of collecting sufficient identity and position related information to assess health \& safety plans in near real-time have been proposed by many researchers. (Riaz at al. 2006) suggest a combination of GPS technologies and MEMS sensors while the authors in (Carbonari et al. 2010) propose an ultra-low power sensor network connected to a central system. In both cases the positional information used are rather coarse, essentially implementing a zoning framework where the presence of workers, equipment and materials in relatively wide areas, usually ranging tenths of square meters, is monitored. On the other hand, complementary approaches, see for example (Bowden et al. 2006), propose a vision where autonomous intelligent systems are able to perform more accurate real-time support to health and safety tasks and hazard prevention by issuing pre-alerts when fatalities are about to occur, by automating dangerous operations and by decreasing human interventions. In these cases, see for example (Teizer et al. 2008), workers' behavioral models are at the basis of intelligent software systems capable of recognizing dangerous situations in real-time and implementing mitigating actions. Of course, this kinds of application require far more accurate tracking information than the zoning framework required by the plan assessment approach, and UWB technology is a good candidate to provide enough accuracy and reliability to support real-time hazard detection. Since the application of UWB to hazard detection systems is still in its early phases, in the rest of this section we will describe the main issues through the discussion of an example of great generality, concerning the implementation of a hardware/software system implementing a virtual fencing facility in construction sites (Carbonari et al. 2011).

In the previous section we have demonstrated that UWB tracking systems can perform very accurate location tracking, with a $30 \mathrm{~cm}$ accuracy, in real construction sites up until the erection of reinforced concrete structures. Instead, a different and more intense configuration for UWB receivers will be needed to perform monitoring when masonry buildings are monitored. In any case, it emerged that UWB tracking is very good for tracking from the beginning of the construction progress at least until structure frames are built and, therefore, that it would be able to support several automated hazard detection tasks like collision avoidance and virtual fencing. It is generally stated (Wang et al. 2004) that a predictive approach is required to properly manage risky situations, since hazard detection should anticipate the real occurrence of an eventual threat by a time span long enough to allow the mitigating action to be carried out. In principle, the high polling frequency of the UWB technology (from 1 to $60 \mathrm{~Hz}$ ) becomes critical should data be provided at a speed rate that allows algorithms to properly manage risky situations by 
providing alerting signals in time. Proactive risk management systems are usually arranged in two levels. At the higher level, see for example (Howden et al. 2003), behavioural models are used to estimate the route that every monitored worker is going to follow and whether this path is expected to lead to risky situations. In this case, the predicting horizon is of the order of minutes. (Teizer at al. 2008) describes a new framework and its algorithms about using frequent positioning data to elicit rapid learning among construction site managers and workers in relation to safety. If accurately collected and in real-time, the location, speed and trajectory of construction resources (e.g. workers, equipment, materials) can lead to important information regarding travel patterns. This information can then be shared among project stakeholders to improve work practices, e.g. the preconditions for safe construction operations. At the lower level, within a predicting horizon of tenths of seconds, the positions of workers are continuously monitored and last minute warnings are sent if actual behaviour may lead to a possible risky situation. (Cheng et al. 2011) and (Teizer et al. 2008) give initial evidence about the potentials of UWB systems in hazard detection, concerning the movement of material and workers' interference applied to this level. Therefore, a more in depth assessment of the UWB technology in supporting the development of hazard detection algorithms is required. The virtual fencing example we are about to discuss moves in this direction. The implementation of virtual fencing logics (Figure 8) is relatively straightforward (Carbonari et al. 2011).

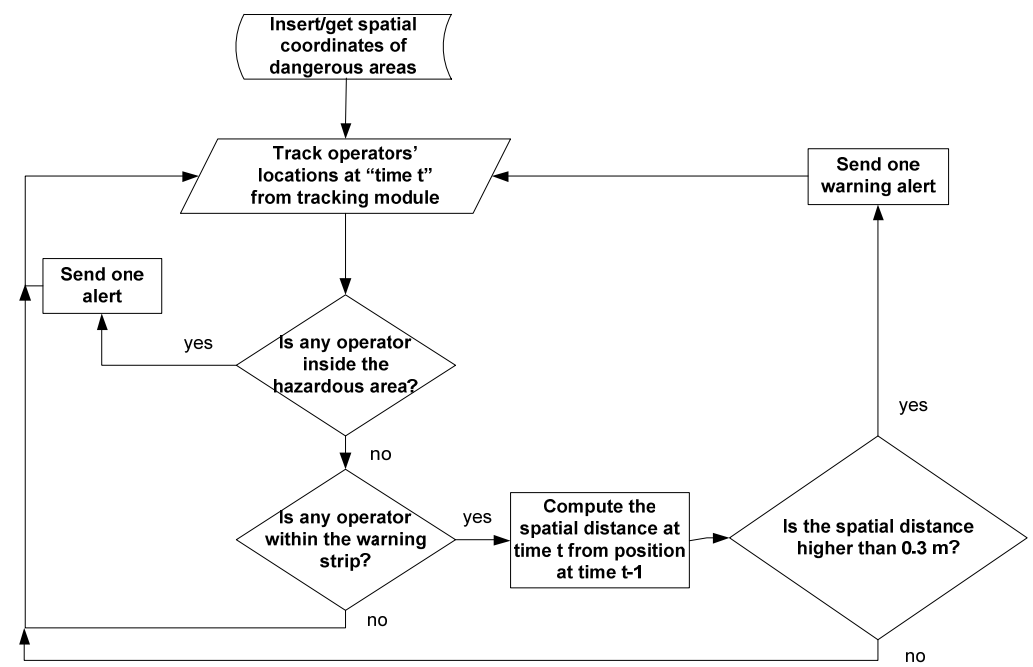

Figure 8. The flow-chart representation of virtual fencing logics.

Each forbidden area is surrounded by a "warning" strip (yellow area) as shown in Figure 9. The width of the warning strips must be determined according to tag frequency and to the speed of average operators. Workers' speed can be assumed to be fixed at $0.5 \mathrm{~m} / \mathrm{s}$, while specific testing is required to determine the most appropriate strip width. This will be further discussed later. The algorithm thus avoids the complexity of having to poll every worker on the construction facility by focusing only on the ones that have entered the 
yellow area. Figure 8 explains a simple virtual fence logic. The algorithm first checks whether an operator has already entered the red dangerous area; if so, a red alarm is sent. If no operator has entered the defined area, no alarms are sent, and the algorithm checks whether any operator has entered the surrounding yellow strip. If so, then the worker's actual position is compared with the previous one (recorded in the previous time step) and a warning alarm is sent only when the distance between the two positions in steps $t$ and $t-1$ is greater than $0.3 \mathrm{~m}$. Otherwise, the entire procedure is repeated. It is clear that tracking operators' movements within the yellow strip is critical for this application.

Some experimental campaigns, carried out by the authors, aimed at evaluating whether a real UWB position tracking system can suit a simple virtual fencing logic, like the one proposed. A number of laboratory experiments were conducted initially in order to assess the general reliability of the UWB system for this task and the width of the yellow warning strip around the red area.
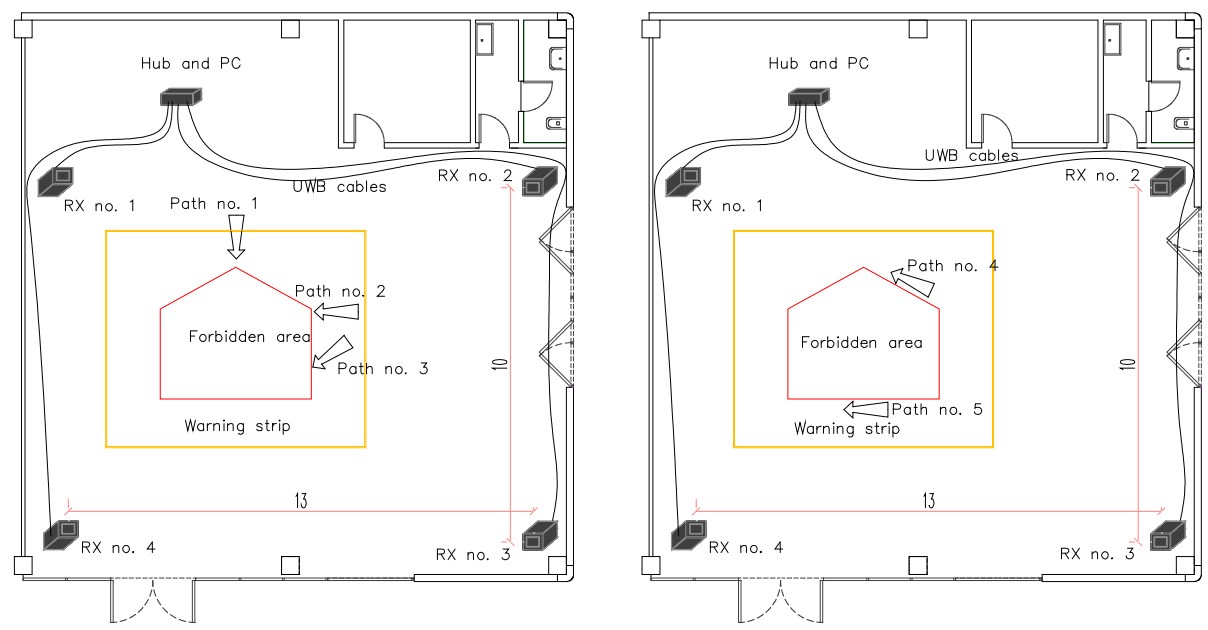

Figure 9. Plant lay-out of the laboratory experiments for the development of the UWB virtual fencing algorithm.

From empirical on-site observations, the workers' average speed was estimated to be around $0.5 \mathrm{~m} / \mathrm{s}$. Hence, in an ideal optimal localization accuracy, considering the polling frequency of the UWB system equal to $1 \mathrm{~Hz}$, a yellow strip around the red area slightly wider than $1 \mathrm{~m}$ should be enough to obtain two resource position samples during area transit. Considering that the system usually does not localize perfectly in phase with moving workers as they cross the region boundary (i.e. in the limit case, the first localization may take place $1 \mathrm{~s}$ after crossing the border line), the width of the yellow strip was increased to $1.5 \mathrm{~m}$. In addition, UWB has a tracking inaccuracy which, even if very low, produces a localization error fluctuating randomly around the average value, even when a worker is in fact still. The estimated position variations of the tracked resource around the mean, generally limited to a 
few tens of centimeters, in rare cases can reach up to 1 meter. This rare, but rather high localization error for the virtual fencing case, can cause critical inaccuracy and produce false or missed alarms. In order to verify the impact of these position errors, some tests were carried out letting one of the crew members (equipped with the $1 \mathrm{~Hz}$ badge tag) repeatedly cross the yellow line drawn $1.5 \mathrm{~m}$ from the border line of the red area and following different paths. The system was not capable of signaling the hazardous event in time in a significant number of cases $(30 \%$ in the worst case). Hence, a statistical autoregressive filtering technique was applied to try to improve localization accuracy, highlighting that a system of order 3 , with weighting coefficients $(0.10,0.20,0.70)$, showed the best performance, providing a good trade-off between signal stability and trajectory prediction. The worst detecting condition occurs when the workers move along parallel paths (Figure $9 b)$. In this case the number of false alarms increase unless more refined checks concerning the actual direction of the workers are carried out. This is the meaning of the dashed box section of the fencing algorithm. At the end, the UWB proved to be sufficiently reliable for further on-site testing. Table 3 reports the number of false alarms registered by the system in the laboratory experiments.

\begin{tabular}{|c|c|c|c|c|c|}
\hline trial & Path no. 1 & Path no. 2 & Path no. 3 & $\begin{array}{c}\text { Path no. 4 } \\
\text { (false Alarms) }\end{array}$ & $\begin{array}{c}\text { Path no. 5 } \\
\text { (false Alarms) }\end{array}$ \\
\hline $\mathbf{1}$ & 0.0 & 0.1 & 0.6 & 1 & 1 \\
\hline $\mathbf{2}$ & 0.0 & 0.2 & 0.4 & 1 & 0 \\
\hline $\mathbf{3}$ & 0.7 & 0.6 & 0.4 & 0 & 1 \\
\hline $\mathbf{4}$ & 0.1 & 0.1 & 0.7 & 0 & 0 \\
\hline $\mathbf{5}$ & 0.3 & 0.0 & 0.3 & 0 & 0 \\
\hline $\mathbf{6}$ & 0.4 & 0.6 & 0.4 & 0 & 0 \\
\hline $\mathbf{7}$ & 0.2 & 0.5 & 0.6 & 1 & 1 \\
\hline $\mathbf{8}$ & 0.4 & 0.4 & 0.4 & 0 & 0 \\
\hline
\end{tabular}

Table 3. Number of false alarms registered during the laboratory experimental campaign.

The field testing validation was performed on a real construction site. The system worked fairly well, reliably providing smooth paths, with a delay determined only by the time required by the UWB system to update the tag's location, and demonstrating the same precision as that shown in the laboratory tests. The assessment of overall system performance was accomplished through three trials which consisted in letting the crew member approach the predefined hazardous area. A red tape was laid out on the ground, along the border line drawn virtually in the GUI windows (Figure 10), around the hazardous zone, where warning signals were expected to be set off. The time when the signal was sent, with respect to the worker's position, was recorded. In conclusion, both the laboratory results and field testing showed that UWB position tracking technology provides the performance that may suit the requirements of real-time hazard detection algorithms. The detailed technical discussion was limited to outdoor construction sites, where UWB localization has been proven accurate enough to provide reliable data for virtual fencing. 

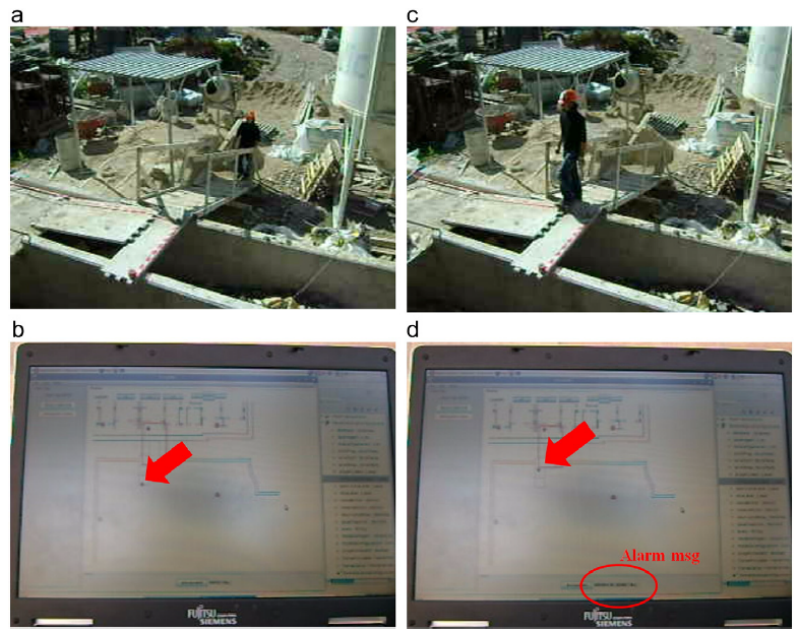

Figure 10. A snapshot of the virtual fencing experiment, conducted in a real construction site. A worker is approaching a critical area $(a, b)$. Signaling occurs before the worker enters the area $(c, d)$.

Despite this almost optimal operational condition, UWB technology demonstrated to be at the lowermost requirement limit (e.g. the $30 \mathrm{~cm}$ accuracy was comparable to the $1.5 \mathrm{~m}$ width of the yellow zone), its extension to indoor environments, which will decrease overall performance, will necessarily require a specific careful UWB lay-out design. This aspect is currently an open research point and requires further investigation.

\section{Real-time work progress tracking}

Building construction is an information intensive process which can easily incur in information overload of the management organization. Efficient management may be hindered by time wasted in information retrieval, by poor and complex information structuring and by delayed communications. Embedded ICT systems for improved information management and the automated control of project performances are currently the foremost frontier of construction project management (Abdelsayed \& Navon 1999), (Navon \& Goldschmidt 2003). By collecting low level field data (e.g. location of workers, materials and facilities) in real-time and inputting them into pattern and process recognition algorithms, these systems promise to support progress control and deviation analysis, improving the human capability of managing large and complex sets of workflow data. Past experiments demonstrated that the tracking of workers and comparisons with projects' baseline can be used to assess activities in progress and related preliminary prototypes, provided an accuracy error lower than $20 \%$ (Navon \& Goldschmidt 2003). Extending this concept to automated activity progress monitoring, successful findings have been reported in the field of earthmoving control (Navon et al. 2004), supply management (Navon \& Berkovich 2006), road construction (Navon \& Shpatnisky 2005). The authors (Carbonari et al. 2011) argue that UWB location tracking data can be effectively applied to automated work sampling. In fact, location tracking data can be used to decompose the presence of workers 
and equipment in the different working area zones versus time and in relation to the trajectories of workers and material and can, in principle, be used to argue the work progress.

Robust custom designed pattern recognition algorithms are then necessary to abstract the activity type and intensity from the raw tracked data and, again, this is still an open research field. To demonstrate how UWB can be applied to work progress estimation consider the following example, excerpted by a construction work tracking campaign led by the authors during the construction of a reinforced concrete frame block of flats. Figure 11 shows some photos of the construction site and Figure 12 details the layout of the building's first floor. Site cast concrete slab erection is the monitored activity illustrated.

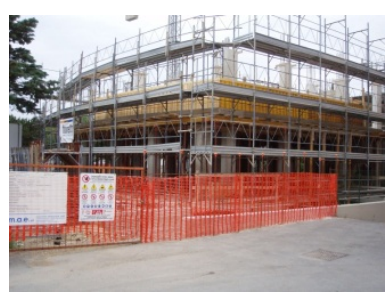

(a)

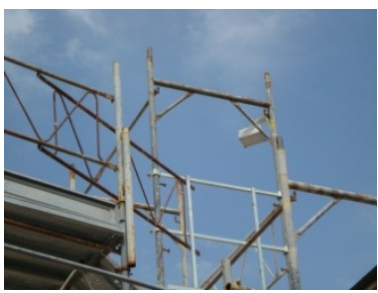

(b)

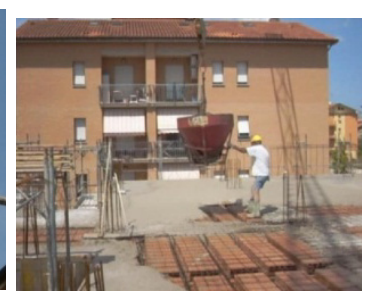

(c)

Figure 11. Pictures of the construction site set-up (a), of the receivers installation in the scaffolding (b) and of the casting phase (c) for the UWB workflow tracking experiment.

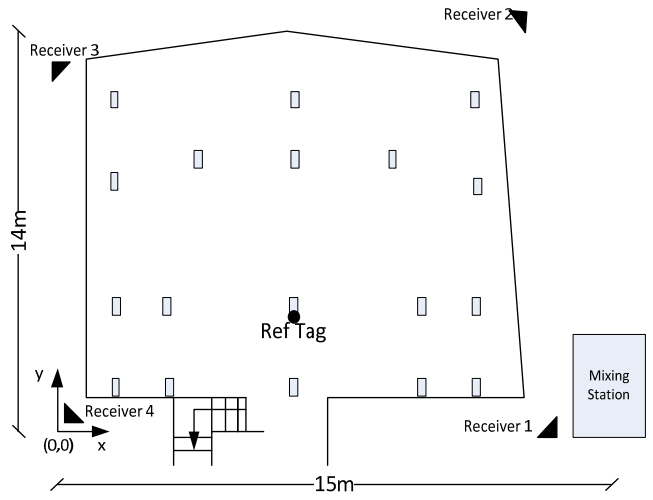

Figure 12. A photo and the plant lay-out of the on-site experiments for the UWB workflow tracking experiment.

One crew out of three was devoted to this task; two working on the site cast concrete slab and one controlling the crane trajectories. The crane moves the crane bucket from the concrete mixing station, placed approximately at referenced position $(15,0)$ to the current concrete pouring place, where it is tended to by one of the two workers, while the other levels and vibrates the liquid concrete poured. Figures 13 shows the crane bucket $X$ and $Y$ coordinates time progress. It can be clearly seen that the $x=15$ peaks correspond to the $y=0$ valleys and that the pattern occurs initially about seven times close to the $x=0$ coordinates, with an increasing trend of the $y$ coordinates. This is easily conceivable as representing the first of concrete 
casting strip, corresponding to the left most side of the floor, as actually occurred. The work then progresses with analogous trends until the crane bucket is stopped in the mixing station area of the construction site. Figure 14 shows the position of the worker on the slab. Even in this case, it can be clearly seen how the worker's position follows the crane bucket's position exactly when the latter is on the casting floor. In fact, the worker is deputed to directing the bucket locally so that the concrete is spread as uniformly as possible during the pouring phase. This is an activity constraint that is correctly and accurately represented by the UWB tracking system. Finally, the number of buckets poured can be easily counted by identifying the crane position peaks at $x=15 \mathrm{~m}$, allowing for a very accurate work progress record. Summarizing, the proposed snapshot of a UWB tracing of a simple construction task shows that activity patterns can be clearly identified through the tracks of workers, materials and equipment. The identity information available from the unique tag identifier, and the relationships that can be drawn straightforwardly with the workflow baselines, represent a very good and reliable basis for implementing advanced work progress tracking systems in construction facilities.

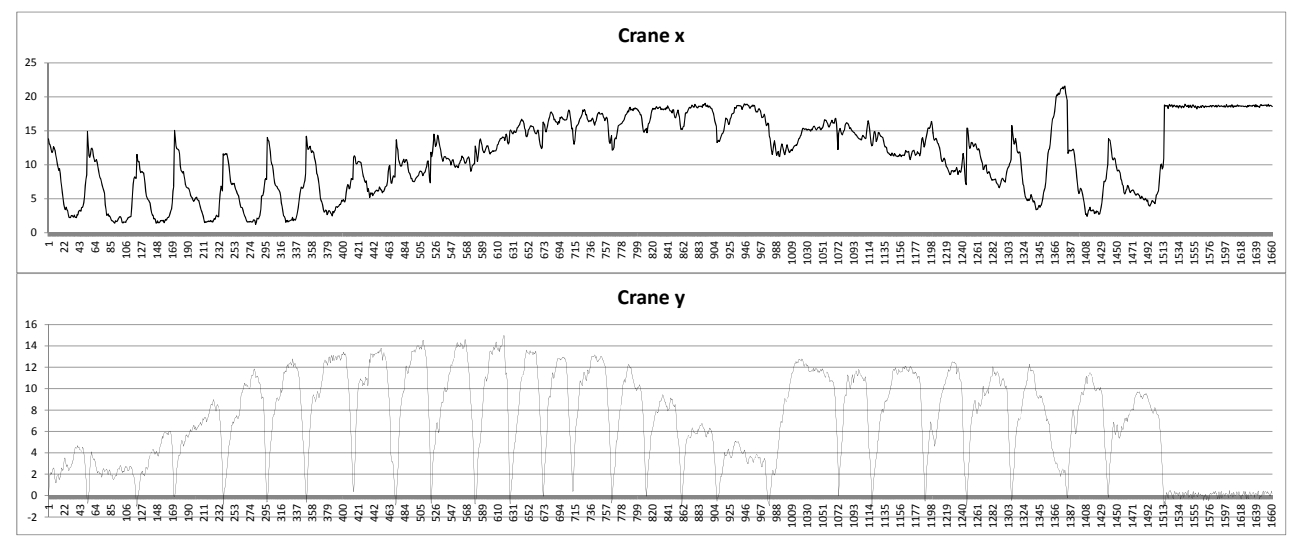

Figure 13. The time progress of the crane bucket $X$ and $Y$ coordinates.

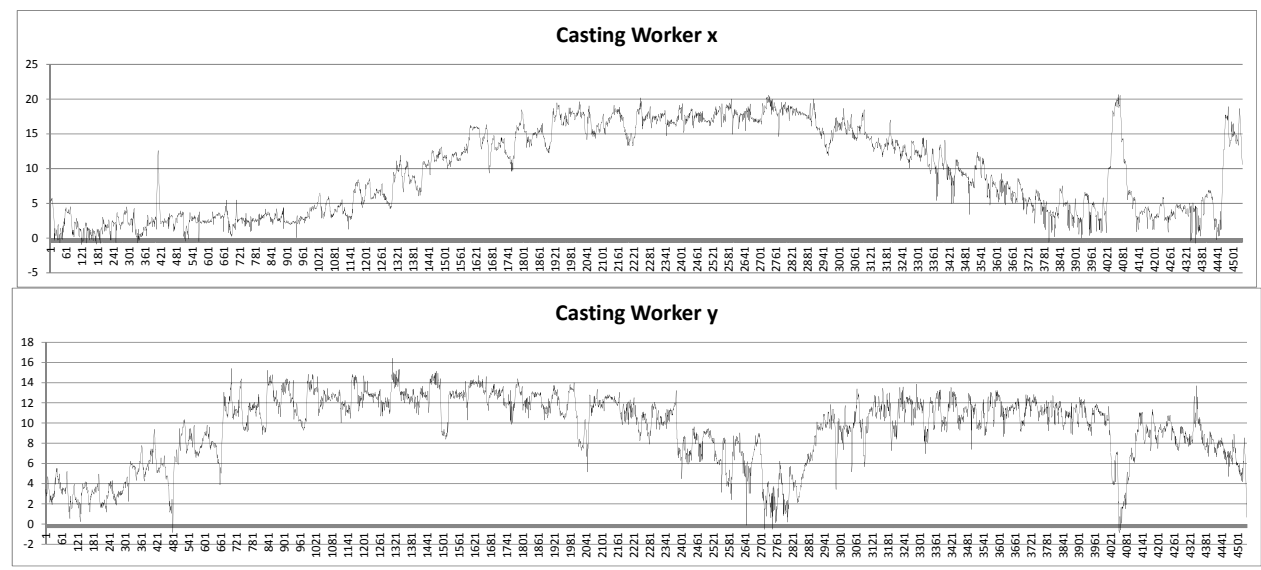

Figure 14. The time progress of the casting worker's $X$ and $Y$ coordinates. 


\section{General design guidelines}

UWB position tracking has demonstrated to be completely reliable in real world construction sites up until the completion of structural frames, providing $30 \mathrm{~cm}$ real-time accuracy. Sections four and five demonstrated that, within this operational boundary, the accuracy provided can suit both the development of advanced systems for work progress estimation and automated systems for health \& safety management as well as real-time hazard risk detection. The UWB indoor tracking capabilities in a construction site boundary installation fade considerably when envelopes and partition walls are raised. In this case, two main design options are available:

- $\quad$ either to deploy a more fine grained UWB arrangement, with receivers installed on the scaffolds of each construction block;

- or to complement the UWB boundary installation with a flexible ultra-low power ZigBee based zoning systems (see introduction).

In the first case, the analysis carried out in section 3 pointed out that in general, UWB is able to pass through a maximum of two heavy walls. Therefore, assuming this as a general design rule for pure UWB implementation, a design procedure can be stated according to the following steps (see Fig. 15a):

1. for each indoor space draw a line of sight form its center to the outside of the building;

2. rotate the line by $360^{\circ}$ and mark the sectors where the number of intersected heavy walls is less than two;

3. the intersections of the sectors drawn for the different indoor spaces define the candidate areas for placing UWB antennas;

4. place receivers so that each indoor space can see at least three receivers through its marked sectors.

Fig. 15a shows the optimized layout obtained with this procedure leading to the increased indoor tracking quality reported in Table 2 of section 3.

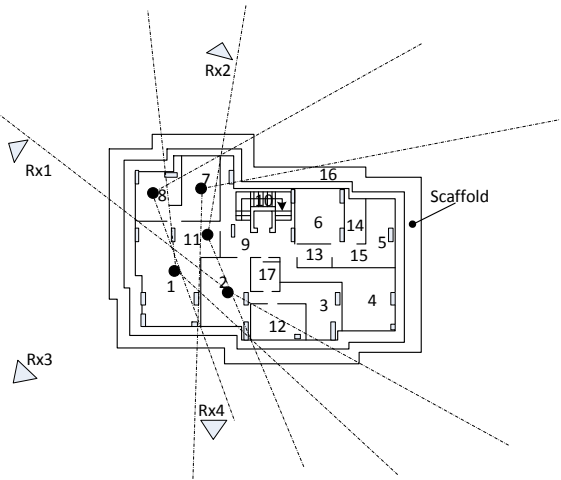

(a)

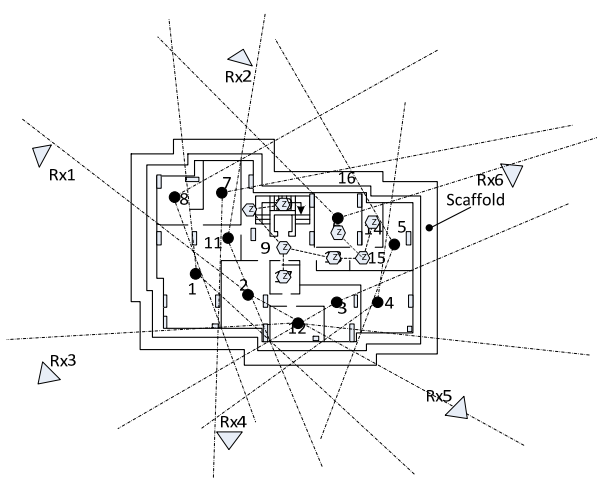

(b)

Figure 15. (a) Optimized receiver layout design for indoor spaces $1,2,3,7,8,11$; (b) mixed mode UWB ZigBee (hexagons) zoning design for mitigating loss of tracking in indoor space 9,10,13,14,15 and 17. 
It may be the case that it's not possible to find a receiver layout providing three suitable receivers for all the indoor spaces of the building under construction. Fig. 15b shows that indoor spaces 9, 10, 13, 14, 15 and 17 cannot be served by any receiver boundary configuration. In this situation a hybrid mode design can be used to mitigate the localized UWB indoor loss of tracking.

The ZigBee zoning systems can be used to assess the presence of the worker in the UWB shadowed zone. Current ultra-low power ZigBee based zoning systems provide enough flexibility to be deployed without any significant impact on the construction site. Despite ZigBee zoning systems cannot provide accurate position tracking, they can still be used to support a number of high level management tasks like work progress tracking or interference monitoring among different construction tasks. Figure 15b shows the hybrid solution schema. The ZigBee network (hexagons) has been deployed to cover the most critical indoor spaces, like number 13 , that cannot be easily served by boundary UWB. Some spaces like space 6 in this example can be served either by increasing the number of UWB receivers or by ZigBee zoning. It's up to the designer defining the most suitable solution. The integration of the UWB and the ZigBee systems can be either basically accomplished by switching to the second system when the first fails providing reliable data or by more complex sensor fusion approaches. However, the hybrid mode tracking position system design and its relationship with high level management tasks is still, at present, an open research issue.

\section{Conclusions}

In this chapter we have discussed the strength and weakness of UWB position tracking technology applied to building construction environments. UWB position tracking has demonstrated to be completely reliable in real world construction sites up until the completion of structural frames, providing support for real time management and health \& safety tasks. The UWB indoor tracking capabilities in boundary installations fade considerably when envelopes and partition walls are raised. In these cases, optimized UWB design with increased number of receiver or hybrid design merging UWB with ZigBee based zoning systems are both possible. In the first case the tracking resolution of the UWB system is maintained at the expense of an increased number of receivers, rising significantly the equipment and installation costs. Anyway it is often the case that UWB boundary installation cannot suit indoor tracking in some spaces whatever number of receivers is used. In that frequent case hybrid mode position tracking design can provide support for high level management tasks. In general, hybrid mode position tracking in construction sites is still an open research issue. More specifically the open issues concern the sensor fusion approach and the relation between the tracking accuracy and the level of support of high level management tasks. Sensor fusion can be as simple as switching between systems in case of tracking failure, or much more complex as merging information at data processing level, developing hybrid TOA - SS algorithms. How optimized performance can be achieved in hybrid frameworks through careful layout design of hybrid sensor systems is a second point that requires investigation and on-site testing. In this chapter we have shown how 
boundary UWB set-ups can be complemented by ZigBee zoning systems deployed in the inner indoor spaces. Nevertheless in these cases some redundancy can be used to increment reliability of the UWB tracking and the robustness of the overall solution. To what extent this can be done is at present not known. Finally the localized lower resolution provided by the ZigBee zoning system may potentially downgrade the support to the high level management tasks. This depends both on the resolution and on the specific lay-out of the tracking solution with respect to the spatial arrangement of the working activities. At present there are not any insights on these advanced design features.

\section{Author details}

Alberto Giretti, Alessandro Carbonari, Massimo Vaccarini

Department of Civil and Building Engineering and Architecture,

Research Team: Building Construction and Automation, Università Politecnica delle Marche, Ancona, Italy

\section{References}

Abderrahim, M.; Garcia, E.; Diez, R. \& Balaguer, C. (2005). A mechatronics security system for the construction site, Automation in Construction, Vol. 14, No. 4, pp.460-466.

Abdelsayed, M. \& Navon, R. (1999). An information sharing, internet based, system for project control, Civil Engineering and Environmental Systems, Vol. 16(3), pp. 211-233.

Behzadan, A., H.; Aziz, Z.; Anumba, C., J.; \& Kamat, V. R. (2008). Ubiquitous location tracking for context-specific information delivery on construction sites, Automation in Construction, 17(6), pp. 737-748.

Blumenthal, J.; Grossmann, R.; Golatowski, F. \& Timmermann, D. (2007). Weighted Centroid Localization in Zigbee-based Sensor Networks, Proceedings of the IEEE International Symposium on Intelligent Signal Processing (WISP 2007), Madrid, Spain.

Bowden, S.; Dorr, A.; Thorpe, T. \& Anumba, C. (2006). Mobile ICT support for construction process improvement, Automation in Construction, 15, pp. $664-676$.

Caffery, J., Jr. (2000). Wireless Location in CDMA Cellular Radio Systems. Boston, MA: Kluwer.

Carbonari, A.; Biscotti, A.; Naticchia, B.; Robuffo, F. \& De Grassi, M. (2010). A management system against major risk accidents in large construction sites, Proceedings of the 27th ISARC, Bratislava, Slovakia.

Carbonari, A.; Giretti, A. \& Naticchia, B. (2011). A proactive system for real-time safety management in construction sites, Automation in Construction, Volume 20, Issue 6, pp. 686-698.

Cheng, M. Y.; Lien, L. C.; Tsai, M., H. \& Chen, W., N. (2007). Open-building maintenance management using RFID technology, Proceedings of the 24th International Symposium on Automation and Robotics in Construction - ISARC 2007, Kochi, India.

Cheng, T.; Venugopal, M.; Teizer, J. \& Vela, P., A. (2011). Performance evaluation of ultra wideband technology for construction resource location tracking in harsh environments, Automation in Construction, Volume 20, Issue 8, pp. 1173-1184. 
Cook, C., E. \& Bernfeld, M. (1970). Radar Signals: An Introduction to Theory and Applications, Academic Press, New York.

Ergen, E.; Akinci, B.\& Sacks, R. (2007). Tracking and locating components in a precast storage yard utilizing radio frequency identification technology and GPS, Automation in Construction, Vol. 16, No. 3, pp.354-367.

European Commission - Directorate-General for Employment: Social Affairs and Equal Opportunities(2008). Causes and circumstances of accidents at work in the EU, Office for Official Publications of the European Communities, Luxembourg, ISBN 978-92-7911806-7.

Fontana, R., J. \& Gunderson, S., J. (2002) Ultra-wideband precision asset location system, Proceedings of 2002 IEEE Conference on Ultra Wideband Systems and Technologies, Baltimore, MD.

Fuller, S.; Ding, Z. \& Sattineni, A. (2002). Case Study: Using the Wearable Computer in the Construction Industry, Proceedings of the 19th ISARC, National Institute of Standards and Technology, Gaithersburg, Maryland, pp. 551-556.

Gajamani, G., K. \& Varghese, K. (2007). Automated project schedule and inventory monitoring using RFID, Proceedings of the 24th International Symposium on Automation and Robotics in Construction - ISARC 2007, Kochi, India.

Gezici, S.; Tian, Z.; Biannakis, G., B.; Kobayashi, H; Molisch, A., F.; Poor, H., V. \& Sahinoglu Z. (2005). Localization via Ultra-Wideband Radios, IEEE Signal Processing Magazine, 70.

Howden, N.; Curmi, J.; Heinze, C.; Goss, S. \& and Murphy, G. (2003). Operational Knowledge Representation: Behaviour Capture, Modelling and Verification, Proceedings of the Eighth International Conference on Simulation Technology and Training (SimTecT '03), Adelaide, Australia.

Khoury, H., M. \& Kamat, V., R. (2000). Evaluation of position tracking technologies for user localization in indoor construction environments, Automation in Construction 18, pp. 444-457.

Navon, R. \& Berkovich, O. (2006). An automated model for materials management and control, Construction Management and Economics, Vol. 24, pp. 635-646,.

Navon, R. \& Goldschmidt, E. (2003). Can Labor Inputs be Measured and Controlled Automatically?, Construction Engineering and Management, July/August issue, pp. 437445.

Navon, R.; Goldschmidt, E. \& Shpatnisky, Y. (2004). A concept proving prototype of automated earthmoving control, Automation in Construction, Vol. 13, pp. 225-239.

Navon, R. \& Shpatnisky, Y. (2005). Filed experiments in Automated Monitoring of Road Construction, Construction Engineering and Management, April issue, pp. 487 - 493.

Poor; H.,V. (1994). An Introduction to Signal Detection and Estimation, 2nd ed., Springer-Verlag, New York.

Qi, Y. \& Kobayashi, H. (2003). On relation among time delay and signal strength based geolocation methods, in Proc. IEEE Global Telecommunications Conf. (GLOBECOM'03), San Francisco, CA, vol. 7, pp. 4079-4083. 
Rebolj, D.; Magdič, A. \& Čuš Babič, N. (2001). Mobile computing in construction. Advances in Concurrent Engineering, Proceedings of the 8th ISPE International Conference on Concurrent Engineering - Research and Applications, California, USA.

Riaz, Z.; Edwards, D., J. \& Thorpe, A. (2006). SightSafety: a hybrid information and communication technology system for reducing vehicle/pedestrian collisions, Automation in Construction, Vol. 15, No. 6, pp. 719-728.

Sahinoglu, Z. \& Catovic, A. (2004). A hybrid location estimation scheme (H-LES) for partially synchronized wireless sensor networks, in Proc. IEEE Int. Conf. Communications (ICC 2004), Paris, France, vol. 7, pp. 3797-3801.

Shimizu, Y. \& Sanada, Y. (2003). Accuracy of relative distance measurement with ultra wideband system, Proc. IEEE Conf. Ultra Wideband Systems and Technologies (UWBST'03), Reston, VA, pp. 374-378.

Teizer, J.; Lao, D.; Sofer, M. (2008). Rapid automated monitoring of construction site activities using Ultra-WideBand, Proceedings of the 24th International Symposium on Automation and Robotics in Construction - ISARC 2007, Kochi, India.

Wang, A., P.; Chen, J., C. \& Hsu, P. L. (2004). Intelligent CAN-based Automotive Collision Avoidance warning system, Proceedings of the 2004 IEEE International Conference on Networking, Sensing \& Control, Taipei, Taiwan. 